\title{
Static versus dynamical mean-field theory of Mott antiferromagnets
}

\author{
G. Sangiovanni, ${ }^{1}$ A. Toschi, ${ }^{1}$ E. Koch, ${ }^{2}$ K. Held, ${ }^{1}$ M. Capone,${ }^{3,4}$ C. Castellani, ${ }^{4}$ O. Gunnarsson, ${ }^{1}$ \\ S.-K. Mo, ${ }^{5}$ J. W. Allen, ${ }^{5}$ H.-D. Kim, ${ }^{6}$ A. Sekiyama, ${ }^{7}$ A. Yamasaki, ${ }^{7}$ S. Suga, ${ }^{7}$ and P. Metcalf ${ }^{8}$ \\ ${ }^{1}$ Max-Planck Institut für Festkörperforschung, Heisenbergstr. 1, D-70569 Stuttgart, Germany \\ ${ }^{2}$ Institut für Festkörperforschung, Forschungszentrum Jülich, 52425 Jülich, Germany \\ ${ }^{3}$ INFM-SMC and Istituto dei Sistemi Complessi, Consiglio Nazionale delle Ricerche, Via dei Taurini 19, I-00185 Roma, Italy \\ ${ }^{4}$ Dipartimento di Fisica Università di Roma "La Sapienza" piazzale Aldo Moro 5, I-00185 Roma, Italy \\ ${ }^{5}$ Randall Laboratory of Physics, University of Michigan, Ann Arbor, Michigan 48109, USA \\ ${ }^{6}$ Pohang Accelerator Laboratory, Pohang 790-784, Korea \\ ${ }^{7}$ Department of Material Physics, Graduate School of Engineering Science, Osaka University, \\ 1-3 Machikaneyama, Toyonaka, Osaka 560-8531, Japan \\ ${ }^{8}$ Department of Physics, Purdue University, West Lafayette, Indiana 47907, USA
}

(Received 2 February 2006; revised manuscript received 26 April 2006; published 31 May 2006)

\begin{abstract}
Studying the antiferromagnetic phase of the Hubbard model by dynamical mean-field theory, we observe striking differences with static (Hartree-Fock) mean field: The Slater band is strongly renormalized and spectral weight is transferred to spin-polaron sidebands. Already for intermediate values of the interaction $U$ the overall bandwidth is larger than in the Hartree-Fock mean field and the gap is considerably smaller. Such differences survive any renormalization of $U$. Our photoemission experiments for Cr-doped $\mathrm{V}_{2} \mathrm{O}_{3}$ show spectra qualitatively well described by dynamical mean-field theory.
\end{abstract}

DOI: 10.1103/PhysRevB.73.205121

PACS number(s): 71.27.+a, 71.20.-b, 79.60.-i

\section{INTRODUCTION}

In recent years, our ability to calculate strongly correlated materials has substantially improved. To this end, one needs to go beyond the conventional local density approximation (LDA). ${ }^{1}$ New methods had to be developed like LDA $+U,{ }^{2}$ where the LDA is supplemented by a local Coulomb interaction $U$ treated in the static Hartree-Fock (HF) mean-field theory, and its sibling LDA+DMFT, 3,4 which employs the more sophisticated dynamical mean-field theory (DMFT). ${ }^{5}$ While it is generally accepted that LDA+DMFT deals more accurately with strongly correlated metals, the simpler $\mathrm{LDA}+U$ is considered to be sufficient for insulators with a large $U,{ }^{4}$ at least in the presence of magnetic or orbital ordering. Indeed, $\mathrm{LDA}+U$ was tailored for such strongly correlated insulators ${ }^{2}$ and is nowadays widely employed to calculate various physical quantities of these.

In our paper, we work out the differences between a static and a dynamical mean-field treatment of long-range-ordered insulators at intermediate to strong Coulomb interactions $U$. For realistic material calculations, this would correspond to $\mathrm{LDA}+U$ and LDA+DMFT. We study these differences, considering the antiferromagnetic (AF) phase of a simple model, the one-band Hubbard model. We point out that at large $U$ dynamic properties and the structure of excited states are strikingly different in Slater (HF) and DMFT antiferromagnets. More specifically the Slater bands are strongly renormalized, most of the spectral weight is transferred to spinpolaron side bands, and the overall bandwidth is proportional to the noninteracting width $W$, as opposed to the $1 / U$ shrinking found in HF theory. We also performed photoemission spectroscopy (PES) experiments for $\mathrm{V}_{2} \mathrm{O}_{3}$ doped with $1.2 \%$ $\mathrm{Cr}$, considering both the $\mathrm{AF}$ and paramagnetic insulators. These experiments confirm that strongly correlated antiferromagnets are not of Slater (HF) type, while they can in many respects be described by DMFT.
The paper is organized as follows: In Sec. II the DMFT results for the antiferromagnetic phase of the Hubbard model are presented and compared to the exact solution of the $t-J$ model in infinite dimensions. In Sec. III we analyze the evolution of the staggered magnetization and spectral function from weak to strong coupling. This allows us to draw some general conclusions about the validity of DMFT and HF theory in describing both ground- and excited-state properties in the antiferromagnetic phase. In Sec. IV the evolution from a Mott antiferromagnet to a paramagnetic Mott insulator with increasing temperature is discussed. The photoemission spectrum of $\mathrm{V}_{2} \mathrm{O}_{3}$ is also presented in both phases, and it is compared to our theoretical calculation. Finally we present our conclusions in Sec. V.

\section{ANTIFERROMAGNETIC PHASE OF THE HUBBARD MODEL}

Several studies have been performed on the AF phase of the Hubbard model, ${ }^{6}$ but here we focus on large values of $U$, which received less attention in the past, and we exploit some technical advances to improve the accuracy of our DMFT calculation so that we can make definite statements not only about the size of the AF gap but also regarding the inner structure of the (Hubbard or Slater) bands below (and above) the gap. In the large- $U$ limit, we can make contact with the $t-J$ model which has been studied in infinite dimensions by Strack and Vollhardt. ${ }^{7}$ We recover the spin-polaron peaks of Ref. 7 which are, however, dispersive in our calculation, an effect occurring in order $D^{2} / U^{2}$ and hence absent in Ref. 7.

The Hubbard Hamiltonian reads

$$
H=-t \sum_{\langle i, j\rangle, \sigma} c_{i \sigma}^{\dagger} c_{j \sigma}+\text { H.c. }+U \sum_{i} n_{i \uparrow} n_{i \downarrow},
$$

where $t$ is the hopping amplitude, $U$ is the Coulomb repulsion, $c_{i \sigma}\left(c_{i \sigma}^{\dagger}\right)$ are annihilation (creation) operators for spin 


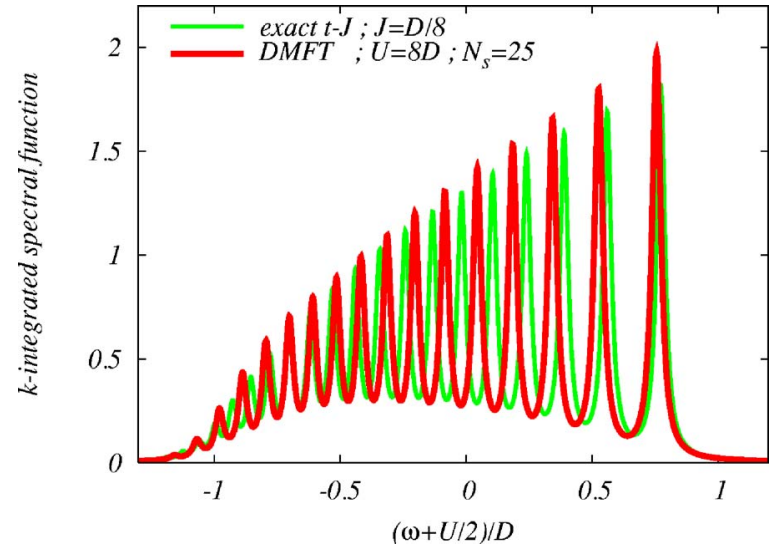

FIG. 1. (Color online) Spectral function of the lower Hubbard band. Red (dark gray) curve: DMFT solution of the Hubbard model for $U=8 D$ and $N_{s}=25$. Green (light gray): $t-J$ model for $J=D^{2} / U$. Both spectra have been plotted using the same Lorentzian broadening.

$\sigma$ electrons on site $i$, and $n_{i \sigma}=c_{i \sigma}^{\dagger} c_{i \sigma}$. We solve the DMFT equations for a semicircular density of states $N(\varepsilon)$ $=\left(2 / \pi D^{2}\right) \sqrt{D^{2}-\varepsilon^{2}}$ with bandwidth $W=2 D$, using exactdiagonalization and Lanczos algorithms at $T=0$ for the associated Anderson impurity model (AIM). A continued-fraction expansion of the DMFT Weiss fields ${ }^{8}$ allows us to obtain reliable spectra in the intermediate-to-large- $U$ region. Moreover, in the AF phase we are able push the number of AIM sites, $N_{s}$, to much larger values than for the paramagnetic phase.

To confirm that we get reliable spectral functions we first consider the large- $U$ limit of the Hubbard model and compare it to the exact solution for the $t-J$ model in infinite dimensions. $^{7}$ The comparison of the $k$-integrated spectral functions is displayed in Fig. 1. In both cases, we show only the negative-frequency part of the spectrum-i.e., the lower Hubbard band centered around $\omega=-U / 2$ (the $x$ axis is shifted correspondingly). Evidently in both cases we find spectra of the same type. We have also verified that the DMFT spectra are almost independent of $N_{s}$, reflecting the fact that, contrary to the case of the paramagnetic insulating phase, in the AF phase the peaks do not originate from the discreteness of the impurity model but have a physical meaning. 7,9

As already observed in Ref. 7 they originate from the fact that a hole moving in the ordered background breaks AF bonds, costing an energy proportional to $n J$, where $n$ is the length of path in the lattice. This string potential gives rise to a set of discrete energy levels with a typical separation proportional to $J^{2 / 3}$. These levels can be interpreted as spinpolaron side peaks ${ }^{10}$ for dispersionless spin waves. ${ }^{11}$ In the $U \rightarrow \infty(J \rightarrow 0)$ limit, these spin-polaron peaks become dense and the lower Hubbard band recovers the shape and width of the noninteracting density of states, with the important difference that the states are incoherent for $U \rightarrow \infty$. In our finite$U$ case, we find that the shift of the peaks in the Hubbard model with respect to the $t-J$ model scales with $1 / U^{2}$. That means we recover the infinite- $U$ limit where the mapping of the Hubbard to the $t-J$ model is exact.

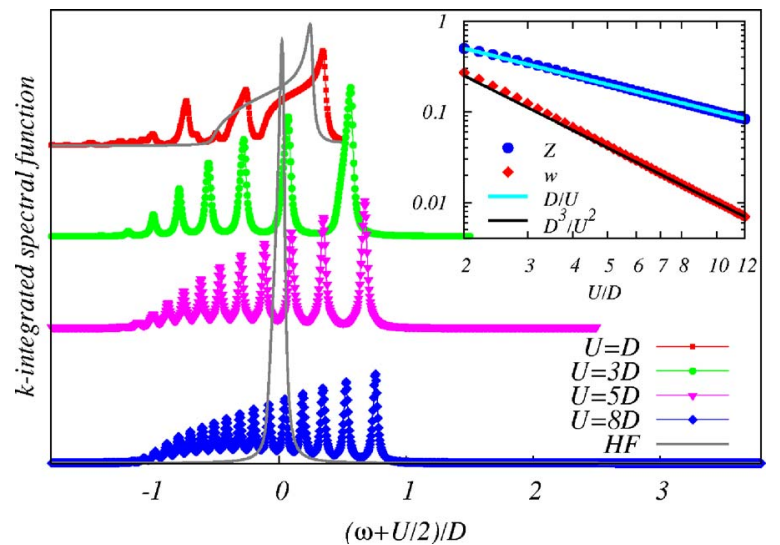

FIG. 2. (Color online) Evolution of the lower Hubbard band with $U / D$. The solid gray lines represent the HF spectrum for $U$ $=D$ and $U=8 D$. In the inset the spectral weight and the quasiparticle dispersion are shown as a function of $U / D$.

\section{STATIC AND DYNAMICAL PROPERTIES FROM WEAK TO STRONG COUPLING}

After having demonstrated the accuracy of our spectra we investigate how the picture changes when $U$ is lower and follow the evolution of our antiferromagnetic solution as a function of $U$.

While for $U=D$ static and dynamical mean-field theories yield, as one expects, similar spectra (see uppermost curve in the main panel of Fig. 2), already for $U=3 D$ the spin-polaron picture characteristic of the $t-J$ model is almost fully developed. This is instead completely missed by the static meanfield theory. The discrepancy between DMFT and HF spectra grows with increasing $U$, as exemplified in the figure for $U$ $=8 D$. The HF spectrum arises from a renormalized singleparticle band with a dispersion decreasing as $1 / U$. In contrast in DMFT the width of the Hubbard band is always of the order of the bare bandwidth even in the limit $U \rightarrow \infty$.

Focusing on the rightmost peak of the Hubbard band, we find that it continuously evolves from the weak-coupling Slater (HF) peak. However, its spectral weight $Z$, directly measured from the spectral density, is dramatically reduced when increasing $U$ since more and more weight is transferred to the spin-polaron sidebands. As it can be seen in the inset of Fig. $2, Z$ scales as $D / U$ for $U \gtrsim 2 D$. This is what is also found for the $t-J$ model; however, the physical nature of the excitation is different. In the $t$ - $J$ model the peaks are dispersionless, ${ }^{7}$ while in the Hubbard model we find, for the peak closest to the Fermi level, a bandwidth $w$ which scales as $D^{3} / U^{2}$ for large $U$ (solid diamonds in the inset of Fig. 2).

An independent argument for the dispersion of this peak is obtained by explicitly looking for the poles of the Green's function. ${ }^{6}$ Averaging over the two sublattices we obtain

$$
G\left(\varepsilon_{k}, \omega\right)=\frac{1}{2}\left[G_{A A}\left(\varepsilon_{k}, \omega\right)+G_{B B}\left(\varepsilon_{k}, \omega\right)\right]=\frac{1}{2} \frac{\zeta_{A}+\zeta_{B}}{\zeta_{A} \zeta_{B}-\varepsilon_{k}^{2}},
$$

where $\varepsilon_{k}$ is the bare band energy, $\zeta_{\alpha}=\omega+\mu-\Sigma_{\alpha}(\omega)$, and $\alpha$ $=A, B$ is a sublattice index. The renormalized dispersion $E_{k}$ is given by the solutions of 


$$
\operatorname{Re} \zeta_{A}(\omega) \zeta_{B}(\omega)-\varepsilon_{k}^{2}=0
$$

with $\varepsilon_{k}$ ranging from $-D$ to $D$.

We label $\omega_{q p}$ the solution for $\varepsilon_{k}=0$. At large $U$ we expand Eq. (3) to linear order and obtain

$$
E_{k}-\omega_{q p}=\frac{1}{\varkappa} \varepsilon_{k}^{2},
$$

where we have defined

$$
\left.\varkappa \equiv \frac{\partial}{\partial \omega} \operatorname{Re} \zeta_{A}(\omega) \zeta_{B}(\omega)\right|_{\omega=\omega_{q p}}
$$

To determine the value of $x$, we consider the Green's function for energies $\omega \sim-U / 2$, which for large $U$ is given by the Green's function of a given sublattice-e.g., $G_{A A}(\omega)$. In this limit, $\Sigma_{B} \approx-\Sigma_{A} \approx U / 2$. Then we find that the quasiparticle weight is given by $Z=-U / \varkappa$.

In particular, considering as an example the case of a hypercubic lattice, the dispersion $E_{k}$ has the following form:

$$
E_{k}-\text { const }=-\frac{Z}{U} \varepsilon_{k}^{2}=-\frac{Z}{U}\left(2 t \sum_{n} \cos k_{n}\right)^{2},
$$

where $k_{n}$ is the wave vector in the $n$th dimension.

The presence of a finite quasiparticle dispersion in the Hubbard model can be understood as an effect of the $O\left(1 / U^{2}\right)$ terms neglected in the mapping to the $t-J$ Hamiltonian. ${ }^{12,13}$ More precisely, in addition to the standard $t-J$ terms, the large- $U$ projection of the Hubbard model leads to spin-flip terms and to second $\left(t_{2}\right)$ and third $\left(t_{3}\right)$ nearestneighbor hopping integrals. Such high-order hoppings contribute to the quasiparticle dispersion even in infinite dimensions and in the specific case of a hole in a Néel state read $t_{2}=2 t^{2} / U$ and $t_{3}=t^{2} / U$, respectively. For a hypercubic lattice these terms give rise to a bare dispersion of the form

$$
\begin{aligned}
\widetilde{\varepsilon}_{k} & =-2 t_{2} \sum_{n \neq m} \cos k_{n} \cos k_{m}-2 t_{3} \sum_{n} \cos 2 k_{n} \\
& =-\frac{1}{U}\left(2 t \sum_{n} \cos k_{n}\right)^{2}-\text { const. }
\end{aligned}
$$

From the results obtained in Ref. 12 for a $t-t^{\prime}-J$ model, we expect for the Hubbard model, in the limit of $t_{2} \ll 2 J$ and $t_{3}$ $\ll 2 J$, a dispersion given by the bare $\widetilde{\varepsilon}_{k}$ of Eq. (7) renormalized by the quasiparticle weight $Z \simeq D / U$. This expectation is in fact confirmed since $Z \widetilde{\varepsilon}_{k}$ coincides with the dispersion given by Eq. (6).

These considerations support the interpretation of our DMFT results as a quasiparticle renormalization of the Slater (HF) peak; the width of this peak shrinks like $D^{2} / U$, and it is further reduced by $Z \simeq D / U$ so that altogether $w \simeq Z D^{2} / U$ $=D^{3} / U^{2}$.

As already mentioned before, a striking difference between the Slater and DMFT spectra is the amplitude of the spectral gap. Since the HF Slater peak is located roughly at the center of the DMFT lower Hubbard band of width of the order of $W$, for large $U$ the spectral gap is reduced from the HF value $U$ to $U-W$. We illustrate this aspect in Fig. 3, where the amplitude of the spectral gap is plotted as a func-

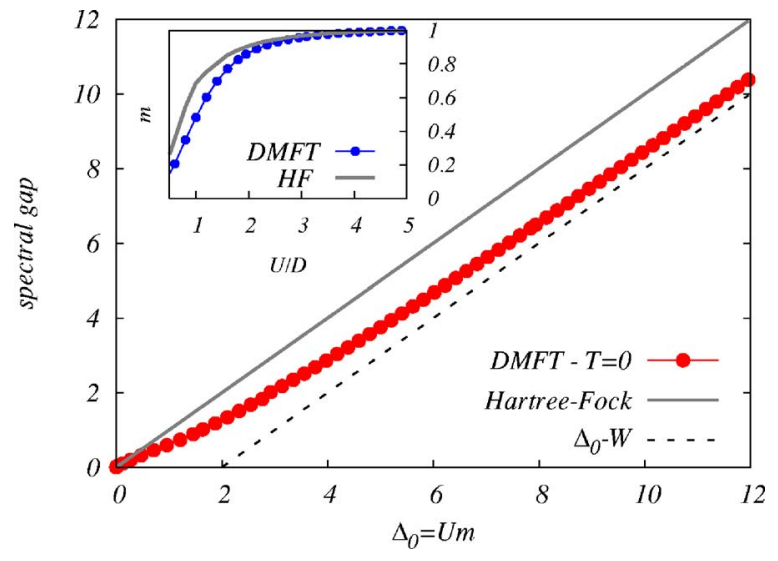

FIG. 3. (Color online) DMFT spectral gap as a function of the AF order parameter $\Delta_{0}=U m$, where $m$ is the staggered magnetization. In the Hartree-Fock case the gap coincides with $\Delta_{0}$ (dashed line). In the inset we compare the DMFT and HF magnetizations as a function of $U$.

tion of the order parameter $\Delta_{0} . \Delta_{0}$ is given by $U$ multiplied by the staggered magnetization $m$, whose behavior is shown in the inset of Fig. 3. The DMFT results indicate that, on the one hand, the staggered magnetization gets closer and closer to the Hartree-Fock value by increasing $U$, while, on the other hand, the spectral gap deviates more and more from the Hartree-Fock prediction $\left(\Delta_{0}\right)$, approaching the asymptotic value of $\Delta_{0}-W$. Thus we have the interesting situation where the two methods give essentially the same magnetization, while there is a sizable difference in the gaps.

Our findings highlight one important point: At large $U$, DMFT and HF theory give the same description of the antiferromagnetic ground state, which is basically the Néel state. On the other hand, the description of excited states is strikingly different between DMFT and HF theory. In HF theory the hole moves in a "rigid" background of Néel spins, while in DMFT, due to quantum fluctuations, the excitations are of a completely different nature: The hole can also move around without paying the double-occupancy cost, with the creation of a spin polaron. Therefore, even if all ground-state quantities ( $m$, kinetic and potential energy, etc.) are basically the same in the two descriptions, excited-state properties (hence spectra) are completely misrepresented by HF theory.

This also means that, within HF theory, it is not possible to simultaneously obtain the correct magnetization and spectral gap with the same value of $U$. This has obvious implications for the use of LDA $+U$ for antiferromagnets (or similarly for orbitally ordered phases) with a large gap.

\section{ANTIFERROMAGNETIC VS PARAMAGNETIC MOTT INSULATOR}

Our DMFT calculations can be extended also to finite temperature. In this case, calculations can only be performed with a lower value of $N_{s}$, but their precision is still sufficient to address a crucial issue of the Mott antiferromagnet: namely, the evolution from a $t-J$ antiferromagnet to a paramagnetic Mott insulator above the Néel temperature. In par- 


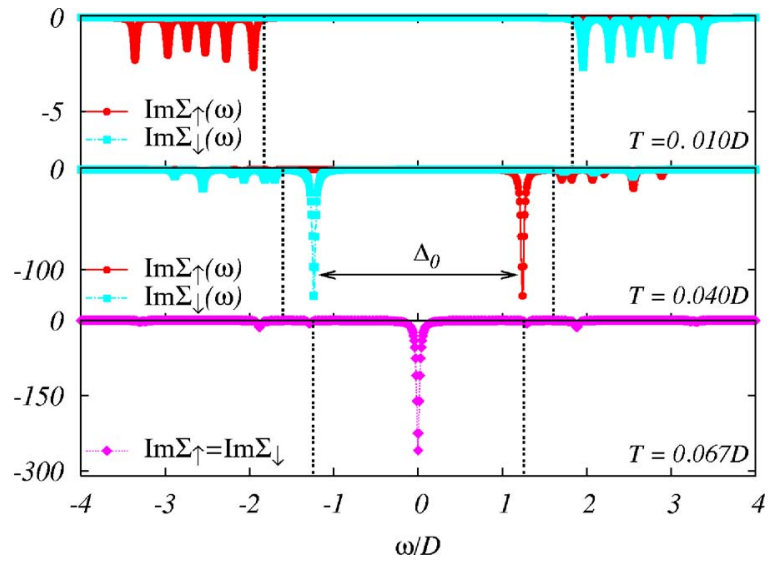

FIG. 4. (Color online) Imaginary part of up and down selfenergy for $U=5 D$, below (two upper panels) and above (lowest panel) the Néel temperature $\left(T_{N}=0.05 D\right)$. The spectral gap is marked by the dashed vertical lines and $\Delta_{0}=U m$.

ticular we are interested in the mechanism ruling the evolution of the system from a fully polarized antiferromagnet with a spectral gap of order $U$ to a Mott insulator with zero magnetization but with a gap of the same order of magnitude. At low $T$, due to the onset of long-range AF order, the opening of the gap is accompanied by a rigid shift in the real parts of the self-energy, whose value is given by the magnitude of the order parameter. The imaginary parts are, in this case, small for both spin species and nonzero only where the corresponding (up or down) spectral weight is present (upper panel of Fig. 4). On the other hand, for $T>T_{N}$, when the order parameter is zero, a Mott gap of order $U$ is found, associated with a $\sim U^{2} / \omega$ peak in the imaginary parts of $\Sigma(\omega)$ at zero frequency, as can be seen in the lowest panel of Fig. 4. How can we go from one situation to the other? By increasing $T$, two peaks in the imaginary parts of $\Sigma(\omega)$ develop inside the spectral gap (indicated by the vertical dashed lines in the figure) and their mutual distance decreases together with the order parameter $\Delta_{0}$ : The two peaks can be viewed as precursor of the $\sim U^{2} / \omega$ behavior in the paramagnetic Mott insulator.

Let us now ask: Are real transition-metal oxides described by static or dynamical mean-field theory? To this end, we performed photoemission measurements for $\mathrm{V}_{2} \mathrm{O}_{3}$ doped with $1.2 \% \mathrm{Cr}^{14}$ The measurement was made at beamline BL25SU of SPring-8 using 500-eV photons. The experimen- tal details are exactly as described previously. ${ }^{15}$ The comparison between theory and experiments is shown in Fig. 5, taking parameters reasonable for $\mathrm{V}_{2} \mathrm{O}_{3}: U=3[\mathrm{eV}]$ and $2 D$ $=2[\mathrm{eV}]$. As we discuss below, the theoretical spectra have been broadened to make contact with the experiment. Both theoretical and experimental spectra are normalized to have the same area. A Shirley-type inelastic background has been removed from the experimental data.

Comparing the experimental data (right panel of Fig. 5) to the spectra we have discussed until now, it is evident that the spiky nature of the latter is absent, or at least basically invisible, in the former. This is evidently due to at least three sources of broadening: the intrinsic experimental broadening and the finite-temperature and finite-dimensionality effects beyond the DMFT. This last source of broadening can, e.g., arise from the coupling to dispersive spin waves. ${ }^{10}$ The size of this effect is hard to estimate for three-dimensional compounds. However, to mimic the overall effect we have plotted in the left panel of Fig. 5 our theoretical results using a broadening of $0.2[\mathrm{eV}]$, larger than the experimental one $(\sim 0.1[\mathrm{eV}])$, in order to include phenomenologically the other effects.

A first observation is that the experimental spectrum in the antiferromagnetic phase has an overall width of the same order of the paramagnet. We have already shown how this is realized in the DMFT spectra, in contrast to the static HF mean-field theory. Theory and experiment qualitatively agree also on the substantial redistribution of spectral weight: Going from the paramagnetic to the AF insulator, an additional peak develops at the upper edge of the lower Hubbard band. This peak is the one we identified as a (quasiparticle-) renormalized Slater band. Besides this peak there is additional spectral weight coming from the broadened spin polaron sidebands. Remarkably, the gap is found to increase when going from the paramagnetic to the AF insulator in both experimental and theoretical spectra. It is worth remarking that this feature does not depend on a particular choice of the value of $U$, since it occurs even in the infinite- $U$ case. ${ }^{16}$ The agreement is less good in one qualitative aspect: the flank at the lower edge of the Hubbard band is almost identical for both phases in experiment whereas they slightly differ in the theoretical calculation. This is likely due to the lack of some realistic features, like an asymmetric density of states and multiorbital effects in our model calculations. This point could be clarified by extending LDA+DMFT calculations from the paramagnetic phase ${ }^{17}$ to $\mathrm{AF} \mathrm{V}_{2} \mathrm{O}_{3}$.
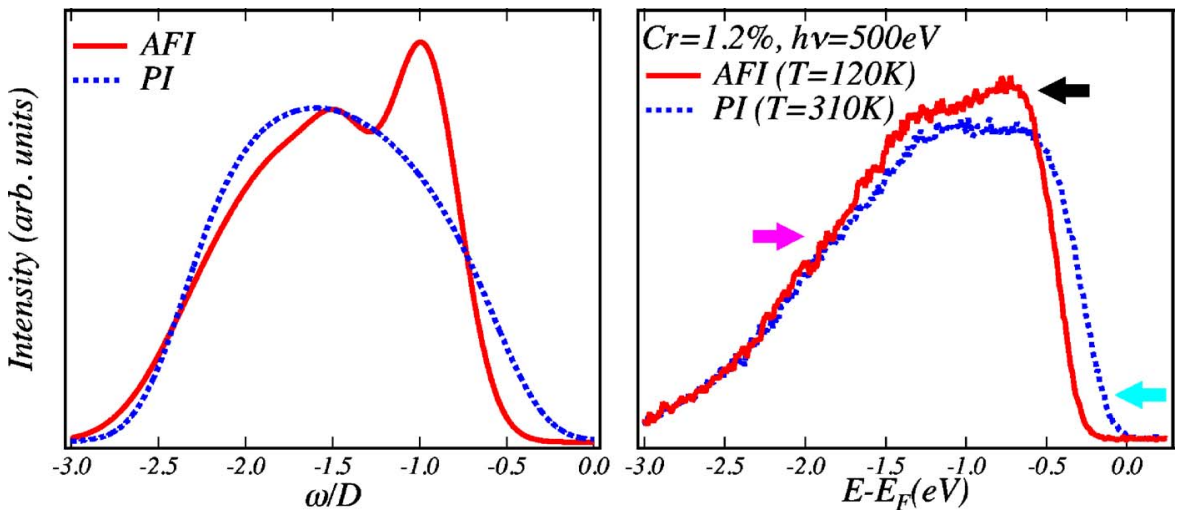

FIG. 5. (Color online) Comparison of theory (left) and PES (right) spectra of the lower Hubbard band in $\mathrm{AF}$ and paramagnetic phases. The black, cyan (light gray), and magenta (dark gray) arrows indicate, respectively, the formation of the (quasiparticle-) renormalized Slater peak, the reduction of the gap in the AF phase, and the qualitative disagreement in the lower edge of the Hubbard band. 


\section{CONCLUSIONS}

In this work we have compared static and dynamical mean-field descriptions of the antiferromagnetic phase of the Hubbard model. We have analyzed the evolution from weak to strong coupling of both ground-state (e.g., magnetization) and excited-state (e.g., spectra) properties. Contrary to the general expectation that HF theory gives a good description of broken-symmetry phases both at weak and strong coupling, we have found that, at large $U$, this is true only for ground-state quantities. The poor description of the excitations given by the static mean-field theory is reflected in a $\mathrm{HF}$ spectrum given only by two unrenormalized $(Z=1)$ Slater bands which shrink as $1 / U$ with increasing $U$. On the contrary, when the excited states are better described, as in DMFT, the Slater bands get strongly renormalized $(Z$ $\simeq D / U)$ and most of the spectral weight is transferred to spin polaron sidebands. Therefore the total width of the Hubbard band stays finite (of order $W$ ) even for large $U$ and the spectral gap is not given by $U$, as in HF theory, rather by $U-W$.

We have also performed PES experiments on Cr-doped $\mathrm{V}_{2} \mathrm{O}_{3}$ in both the antiferromagnetic and paramagnetic phases. We have found a qualitative agreement with the DMFT cal- culations, in the formation of the renormalized Slater peak, in the overall width of the band (of order $W$ rather than $D / U)$, and also in the shrinking of the gap when going from the paramagnetic to the antiferromagnetic phase.

In conclusion, we demonstrated with our DMFT calculation and by comparing theory with experiments that one has to be careful when applying static mean-field theories, like $\mathrm{LDA}+U$, for calculating physical properties of insulators with long-range order. Adjusting $U$ to get the correct gap, one obtains wrong estimates of the magnetization, or the energy, and vice versa. These problems can be overcome by employing dynamical mean-field theory.

\section{ACKNOWLEDGMENTS}

This work was supported by the Emmy-Noether program of the Deutsche Forschungsgemeinschaft (A.T., K.H.), by the U.S. NSF (Grant No. DMR-03-02825), by a Grant-in-Aid for COE Research (No. 10CEW2004), and by MIUR PRIN Cofin 2003. G.S. and M.C. acknowledge the hospitality of Forschungszentrum Jülich. Calculations were performed on the Jülich JUMP computer under Grant No. JIFF22.
${ }^{1}$ W. Kohn and L. J. Sham, Phys. Rev. 140, A1133 (1965); R. O. Jones and O. Gunnarsson, Rev. Mod. Phys. 61, 689 (1989).

${ }^{2}$ V. I. Anisimov, J. Zaanen, and O. K. Andersen, Phys. Rev. B 44, 943 (1991); V. I. Anisimov, F. Aryasetiawan, and A. I. Lichtenstein, J. Phys.: Condens. Matter 9, 767 (1997).

${ }^{3}$ V. I. Anisimov, A. I. Poteryaev, M. A. Korotin, A. O. Anokhin, and G. Kotliar, J. Phys.: Condens. Matter 9, 7359 (1997); A. I. Lichtenstein and M. I. Katsnelson, Phys. Rev. B 57, 6884 (1998).

${ }^{4}$ K. Held et al., Psi-k Newsletter \#56 5 (2003) [psi-k.dl.ac.uk/ newsletters/News_56/Highlight_56.pdf]; G. Kotliar, S. Y. Savrasov, K. Haule, V. S. Oudovenko, O. Parcollet, and C. A. Marianetti, cond-mat/0511085, Rev. Mod. Phys. (to be published); K. Held, cond-mat/0511293 (unpublished).

${ }^{5}$ W. Metzner and D. Vollhardt, Phys. Rev. Lett. 62, 324 (1989); A. Georges, G. Kotliar, W. Krauth, and M. Rozenberg, Rev. Mod. Phys. 68, 13 (1996).

${ }^{6}$ M. Jarrell, Phys. Rev. Lett. 69, 168 (1992); M. Fleck, A. I. Liechtenstein, A. M. Oles, L. Hedin, and V. I. Anisimov, ibid. 80, 2393 (1998); R. Zitzler, T. Pruschke, and R. Bulla, Eur. Phys. J. B 27, 473 (2002); T. Pruschke and R. Zitzler, J. Phys.: Condens. Matter 15, 7867 (2003).

${ }^{7}$ R. Strack and D. Vollhardt, Phys. Rev. B 46, 13852 (1992).

${ }^{8}$ Q. Si, M. J. Rozenberg, G. Kotliar, and A. E. Ruckenstein, Phys.
Rev. Lett. 72, 2761 (1994).

${ }^{9}$ W. F. Brinkman and T. M. Rice, Phys. Rev. B 2, 1324 (1970); L. N. Bulaevskii, E. L. Nagaev, and D. I. Khomskii, Zh. Eksp. Teor. Fiz. 54, 1562 (1968) [Sov. Phys. JETP 27, 836 (1968)].

${ }^{10}$ K. J. von Szczepanski, P. Horsch, W. Stephan, and M. Ziegler, Phys. Rev. B 41, 2017 (1990); G. Martínez and P. Horsch, Phys. Rev. B 44, 317 (1991).

${ }^{11}$ E. Cappelluti and S. Ciuchi, Phys. Rev. B 66, 165102 (2002).

${ }^{12}$ A. Schiller, P. Kumar, R. Strack, and D. Vollhardt, Phys. Rev. B 51, 8337 (1995).

${ }^{13}$ A. Auerbach, Interacting Electrons and Quantum Magnetism (Springer, Berlin, 1994).

${ }^{14}$ Our results have been mentioned before in J. W. Allen, Solid State Commun. 123, 469 (2002); J. Phys. Soc. Jpn. 74, 34 (2005).

${ }^{15}$ S.-K. Mo, H.-D. Kim, J. W. Allen, G.-H. Gweon, J. D. Denlinger, J.-H. Park, A. Sekiyama, A. Yamasaki, S. Suga, P. Metcalf, and K. Held, Phys. Rev. Lett. 93, 076404 (2004).

${ }^{16}$ W. Metzner, P. Schmit, and D. Vollhardt, Phys. Rev. B 45, 2237 (1992).

${ }^{17}$ K. Held, G. Keller, V. Eyert, D. Vollhardt, and V. I. Anisimov, Phys. Rev. Lett. 86, 5345(2001); G. Keller, K. Held, V. Eyert, D. Vollhardt, and V. I. Anisimov, Phys. Rev. B 70, 205116 (2004). 